\title{
Study on the Detection Method of Nitrosamines in Livestock Food
}

\author{
Hou Xiaolin ${ }^{1 *}$ \\ ${ }^{1}$ Qingdao Institute of Technology, Qingdao, Shandong, 266300, China
}

\begin{abstract}
In view of the complex and cumbersome problems in the detection of nitrosamines in the past, this article optimizes the detection method of nitrosamines in livestock food and establishes a more complete detection method of nitrosamines. In this method, the solid alkali heat treatment method is used instead of the national standard method to pre-treat the sample. Then, activated carbon column solid phase extraction method is used for detection, and higher precision and recovery rate are obtained, which proved that the detection method can be popularized and applied in similar detection work.
\end{abstract}

\section{Introduction}

In the processing of livestock food, a certain amount of nitrite is usually added. Although it can inhibit bacteria and maintain the color and flavor, it will also react with the biogenic amines contained in the livestock food to form nitramine. Nitrosamines are heterocyclic carcinogens with strong toxicity. Therefore, in addition to taking measures to reduce the content of nitrosamines, accurate detection of the content of nitrosamines in livestock foods also plays an important role in controlling the quality of livestock foods. Therefore, this article does further research on the detection method of nitrosamines.

\section{An overview of nitrosamines and their pretreatment methods}

Nitrosamine compounds generally have an $\mathrm{N}-\mathrm{N}=\mathrm{O}$ structure, and there are many types of groups connecting this structure. Therefore, there are many types of nitrosamine compounds [1-2]. And this kind of compound is widespread in livestock food. Nitrosamine compounds mainly exist in the form of N-dimethylnitrosamine, N-diethylnitrosamine and N-nitrosodipropylamine. Among them, N-dimethylnitrosamine is the most common, and its volatility and toxicity are higher than other compounds [3]. Relevant studies have shown that these compounds have extremely strong toxicity and carcinogenicity. They mainly damage the digestive system of the human body and can even cause carcinogenic effects on offspring animals through the placenta. According to relevant national standards, the limit of nitrosamine compounds in livestock food is $3 \mu \mathrm{g} / \mathrm{kg}$. This standard is also an important standard in food production and testing. On this basis, various analysis and detection technologies for nitrosamines have also been continuously developed and innovated [4-5].

Since nitrosamines in food cannot be directly quantitatively detected, the food samples must be pre-treated before they can be detected to eliminate the influence of matrix interferences and improve the accuracy of analysis and detection [6]. At present, the pretreatment of food samples is mostly carried out by solid phase extraction technology. This method uses various polymer fiber materials as extraction needles to adsorb nitrosamines, and then under rapid heating conditions, the nitrosamines are desorbed to the chromatographic column for separation. Due to the continuous advancement of livestock food processing technology and strict food safety laws and regulations in recent years, the content of nitrosamines is also declining, and other components in the food may have a certain impact. Therefore, it is still necessary to choose a more appropriate treatment method according to the actual situation [7].

\subsection{Study on the method of Nitrosamine in livestock food by solid phase extraction}

In the previous pretreatment methods, lye treatment is mainly used. Although this method effectively simplifies the complicated steps in the traditional national standard method, since dichloromethane must be used for liquid phase extraction, complicated subsequent treatment steps are still required. For this reason, in this experiment, using the characteristics of some activated carbons to efficiently adsorb nitrosamine compounds, the activated carbon solid phase extraction method is used to detect nitrosamines to achieve the purpose of one-step processing and high-efficiency detection. 


\subsection{Experimental materials and experimental equipment}

The experimental materials include livestock food samples and reagents. The livestock food samples are mainly ham and bacon purchased in the market. The experimental reagents include methylene chloride, methanol, barium hydroxide, and nitrosamine standard solutions.

The experimental equipment includes a high-speed refrigerated centrifuge, a mixer, a gas chromatography-mass spectrometer, a nitrogen blow dryer, activated carbon, a solid phase extraction device and a drying box.

\subsection{Preparation of standard solution}

It needs to dilute the standard solution with methanol to obtain an internal standard mixed solution. It can dilute with dichloromethane to obtain standard working solutions of different concentrations.

\subsection{Sample pretreatment}

It needs to cut a certain amount of sample into small pieces and mix it, place it in a centrifuge tube, add the internal standard mixed solution and let it stand to ensure that the sample has fully absorbed the internal standard mixed solution. Then it can add barium hydroxide and an appropriate amount of distilled water, tighten the centrifuge tube, and place it in an oven to dry. After drying for a certain period of time, it can use a mixer for mixing, and then put it in an oven for treatment, and then perform high-speed centrifugation treatment. The supernatant obtained after the treatment is stored in a suitable environment for later use.

It should place the Sep-pak®plus AC-2 cartridge on the solid phase extraction device, and then connect it to the sample tube with glass wool at the bottom. The Sep-pak $($ plus AC-2 cartridge is activated with dichloromethane, methanol and water in sequence. After the activation treatment is completed, the supernatant obtained by centrifugation is loaded on the small column, and the flow rate of the sample solution is controlled by a vacuum pump. The flow rate is generally controlled at 1-3 drops per second. After all the sample solution has flowed through the Sep-pak ${ }^{\circledR}$ plus AC-2 cartridge, it can continue to run the vacuum pump. After a period of time, the operation is stopped, and a small amount of dichloromethane is used to elute the small column, and the eluted liquid is collected in a centrifuge tube. And at room temperature, it can use a nitrogen dryer for drying treatment, and then filter through a filter membrane, and place the filtered product in a sample bottle for testing.

\subsection{Testing conditions}

\subsubsection{Chromatographic conditions}

The specifications of the chromatographic column are $30 \mathrm{~m} \times 250 \mu \mathrm{m} \times 0.25 \mu \mathrm{m}$. The initial temperature $\mathrm{i}$ set to $35^{\circ} \mathrm{C}$, and then increases to $90^{\circ} \mathrm{C}$ at a rate of $10^{\circ} \mathrm{C}$ per minute, and then to $240^{\circ} \mathrm{C}$ at a rate of $30^{\circ} \mathrm{C}$ per minute, and maintains for about 6 minutes. The chromatographic column uses high-purity $\mathrm{He}$ gas, and the sample is injected in a constant flow mode.

\subsubsection{Mass spectrometry conditions}

The mass spectrometer uses an electron bombardment ion source, the ion source temperature is set at $230^{\circ} \mathrm{C}$, and the multiple reaction monitoring mode is used for detection.

\section{Experimental results and discussion}

\subsection{Analysis of pre-processing conditions}

In order to eliminate the influence of fat in livestock food, it is usually necessary to use lye treatment to saponify fat. Since the sodium hydroxide solution used in the past would make it difficult to separate and clarify the extract, the solid barium hydroxide is used for treatment in this experiment. The use of $\mathrm{Ba} 2+$ to denature and precipitate proteins can promote better separation and clarification of the extract, which is conducive to subsequent purification and enrichment steps, and adding in solid form also has the advantage of convenient experimental operations.

According to previous experiments, barium hydroxide solid may block the small column when the dosage is too high, so $0.9 \mathrm{~g}$ is appropriate. At the same time, too long heating time may cause browning of the extract, and the centrifuge tube is also prone to damage under this condition. Therefore, $80^{\circ} \mathrm{C}$ is used for heating.

\subsection{Selection of H MS conditions}

The main function of the chromatographic column used in this experiment is to effectively separate the co-elution of nitrosamine compounds and other components. In order to avoid the interference caused by the qualitative analysis and quantitative analysis in the multiple reaction monitoring mode, otherwise it will cause the secondary ions in the nitrosamine compound to be interfered by the co-elution and cause signal abnormalities.

\subsection{Sample analysis results}

According to the experimental results, the 8 nitrosamine compounds contained in this sample all have a good linear relationship within the investigated concentration range. The results are shown in table 1. 
Table 1. Regression equation, detection limit and quantitative limit of nitrosamines

\begin{tabular}{|c|c|c|c|c|}
\hline Analyte & $\begin{array}{c}\text { Regression } \\
\text { equation }\end{array}$ & $\begin{array}{c}\text { Correlation } \\
\text { coefficent }\left(\mathrm{R}^{2}\right)\end{array}$ & $\begin{array}{c}\text { Detection limit } \\
(\mu \mathrm{g} / \mathrm{kg})\end{array}$ & $\begin{array}{c}\text { Quantitative limits } \\
(\mu \mathrm{g} / \mathrm{kg})\end{array}$ \\
\hline NDMA & $\mathrm{y}=1.211 \mathrm{x}+0.012$ & 0.9994 & 0.09 & 0.33 \\
\hline NMEA & $\mathrm{y}=0.571 \mathrm{x}+0.003$ & 0.9996 & 0.05 & 0.27 \\
\hline NDEA & $\mathrm{y}=0.298 \mathrm{x}-0.001$ & 0.9998 & 0.04 & 0.13 \\
\hline NDPA & $\mathrm{y}=1.552 \mathrm{x}-0.002$ & 0.9992 & 0.19 & 0.63 \\
\hline NDBA & $\mathrm{y}=2.256 \mathrm{x}-0.019$ & 0.9993 & 0.08 & 0.29 \\
\hline NPIP & $\mathrm{y}=1.254 \mathrm{x}+0.010$ & 0.9991 & 0.21 & 0.70 \\
\hline NPYR & $\mathrm{y}=0.357 \mathrm{x}+0.012$ & 0.9990 & 0.04 & 0.11 \\
\hline NMOR & $\mathrm{y}=3.594 \mathrm{x}-0.032$ & 0.9999 & & 0.36 \\
\hline
\end{tabular}

(Among them, the linear range is $1-200 \mathrm{ng} / \mathrm{mL}$ )

In this experiment, the internal standard objects are marked by the stable is ot ope labeling method, and the results are shown in table 2 .

Table 2. Recovery rates of nitrosamine compounds in food samples from livestock

\begin{tabular}{|c|c|c|c|}
\hline Sample & Analyte & Add a level $(\mu \mathrm{g} / \mathrm{kg})$ & Percent recovery $(\%)$ \\
\hline \multirow{8}{*}{ Ham } & NDMA & $0.3 ; 3 ; 10$ & $118.6 ; 101.4 ; 108.1$ \\
\hline & NMEA & $0.3 ; 3 ; 10$ & $63.2 ; 102.1 ; 108.2$ \\
\hline & NDEA & $0.3 ; 3 ; 10$ & $73.3 ; 101.4 ; 118.7$ \\
\hline & NDPA & $0.3 ; 3 ; 10$ & $68.3 ; 82.9 ; 102.3$ \\
\hline & NMOR & $0.3 ; 3 ; 10$ & $79.1 ; 109.2 ; 122.1$ \\
\hline & NPIP & $0.3 ; 3 ; 10$ & $89.5 ; 103.4 ; 116.2$ \\
\hline & NPYR & $0.3 ; 3 ; 10$ & -; 83.4; 84.6 \\
\hline & NDBA & $0.3 ; 3 ; 10$ & $65.1 ; 63.0 ; 61.2$ \\
\hline \multirow{8}{*}{ Bacon } & NDMA & $0.3 ; 3 ; 10$ & $97.2 ; 95.3 ; 105.1$ \\
\hline & NMEA & $0.3 ; 3 ; 10$ & $78.4 ; 83.4 ; 103.7$ \\
\hline & NDEA & $0.3 ; 3 ; 10$ & $72.0 ; 115.9 ; 124.2$ \\
\hline & NDPA & $0.3 ; 3 ; 10$ & $75.6 ; 84.2 ; 93.8$ \\
\hline & NMOR & $0.3 ; 3 ; 10$ & $65.0 ; 71.3 ; 84.1$ \\
\hline & NPIP & $0.3 ; 3 ; 10$ & $124.0 ; 73.1 ; 84.2$ \\
\hline & NPYR & $0.3 ; 3 ; 10$ & $-; 77.0 ; 118.3$ \\
\hline & NDBA & $0.3 ; 3 ; 10$ & $107.2 ; 64.1 ; 61.0$ \\
\hline \multirow{8}{*}{$\begin{array}{c}\text { Smoked } \\
\text { meat }\end{array}$} & NDMA & $0.3 ; 3 ; 10$ & $93.7 ; 116.3 ; 104.0$ \\
\hline & NMEA & $0.3 ; 3 ; 10$ & $61.8 ; 77.1 ; 74.2$ \\
\hline & NDEA & $0.3 ; 3 ; 10$ & $85.2 ; 84.4 ; 99.8$ \\
\hline & NDPA & $0.3 ; 3 ; 10$ & -; $66.1 ; 74.4$ \\
\hline & NMOR & $0.3 ; 3 ; 10$ & -; 64.3; 75.2 \\
\hline & NPIP & $0.3 ; 3 ; 10$ & $-; 124.2 ; 115.6$ \\
\hline & NPYR & $0.3 ; 3 ; 10$ & -; 69.3; 85.9 \\
\hline & NDBA & $0.3 ; 3 ; 10$ & -; 90.8; 63.9 \\
\hline
\end{tabular}


('-' in the table indicates that the background value is much greater than the added value and is therefore not calculated)

According to the data in table 2 , it can be seen that for the detection of nitrosamines in samples, the recovery rate is relatively high and the overall precision is good, which proves that this method can be effectively used for the detection of nitrosamines in livestock food.

\section{Conclusion}

In this experiment, a new detection method is used to detect nitrosamine compounds in livestock food. The test results show that the test method is not only easy to operate, time-saving and labor-saving, but also better optimization of the existing equipment parameters. The experimental results also show that the method has high precision and recovery rate. Using this method may also cause a small amount of nitrosamine compounds to be lost during the heating process. For this reason, the pre-processing method still needs to be further optimized.

\section{References}

1. Li Li, Wang Yuchuan, Li Shuo, et al. Research progress of nitrosamine detection technology in cosmetics[J]. Chinese Pharmacist, 2021, 24(03): 553-557.

2. Liang Xiuqing, Yang Ying, Zhang Hongxia, et al. Research progress in detection technology of volatile N-nitrosamine compounds in meat products[J]. Meat Research, 2021, 35(01): 98-104.

3. Hou Jing, Lu Yuepeng, Chen Dan, et al. Rapid determination of $\mathrm{N}$-dimethylnitrosamine in meat products using GC-MS[J]. Food Industry, 2020, 41(12): 298-300.

4. Cai Shu, Zhang Rongrong, Deng Feiyan. Discussion on the application of high performance liquid chromatography in food detection[J]. Modern Food, 2020(23): 123-125.

5. Lv Shumei. The application of high performance liquid chromatography (HPLC) in the detection of food additives[J]. Food Safety Guide, 2020(30): 166-167.

6. Huang Zhoumei, Ma Hui, Li Zhanming, et al. Research progress in $\mathrm{N}$-nitrosamine detection methods in food $[\mathrm{J}]$. Journal of Food Safety and Quality Inspection, 2020, 11(19): 6892-6900.

7. Zhang Jianbin, Ma Lizhen, Zhang Tian, et al. Establishment of 9 methods for determination of $\mathrm{N}$-nitrosamines in meat products[J]. Chinese Journal of Food Science, 2020, 20(05): 276-282. 